Der gefundene Widerspruch zwischen BSG und BAG wirft die Frage auf, ob die individualisierende Streikeindämmung durch das BSG Bestand haben wird. Zunächst einmal ist dem vorschnellen Schluß entgegenzutreten, als könne das ökonomische und politische System einen solchen Widerspruch nicht dulden, als müsse es ihn durch Rechtsprechungs- oder Gesetzesangleichung auslöschen. Im Gegenteil, dieser Widerspruch kann in einer relativen gesellschaftlichen Ruhelage selbst wieder Bestandteil dieses Systems sein, indem eben genau das widersprüchliche Nebeneinander von reprimierendem Arbeitskampfrecht und großzügigem sozialem Folgerecht Pazifizierungsinstrument wird. Allerdings wird eine solche Gleichzeitigkeit widersprüchlicher Streikeindämmungsstrategien immer nur eine gesellschaftliche Schwebelage markieren. Würde die Bundesrepublik auf eine Phase politisch-sozialer Auseinandersetzungen zutreiben, dann würde diesem Schwebezustand wahrscheinlich der materielle Boden entzogen. Denn einerseits wäre dann die Eskalation da, die das BSG zu vermeiden sucht, so daß seine individualisierende Rechtsprechung jedenfalls nicht mehr unter jenem Gesichtspunkt zu rechtfertigen wäre. Zum anderen geriete bei einer solchen gesellschaftspolitischen Zuspitzung die Arbeitslosenversicherung viel stärker als bisher - schon aus finanziellen, darüber hinaus aber auch politischen Gesichtspunkten - in den Sog einkommenspolitischer und allgemeinpolitischer Notwendigkeiten, dem sich das BSG wahrscheinlich kaum entziehen könnte. Mit einiger Sicherheit würde das Bundessozialgericht dann auf die Konfrontationslinie des Bundesarbeitsgerichts einschwenken.

Ulrich Mückenberger

\title{
Zur Mitbestimmungsdiskussion in Großbritannien
}

$\mathrm{Zu}$ Jahresbeginn 1977 legte eine staatliche Untersuchungskommission unter dem Vorsitzenden Lord Bullock ihren Bericht' mit konkreten Vorschlägen zur Demokratisierung privatwirtschaftlicher Unternehmen dem Parlament vor. Bereits der vorgegebene Forschungsauftrag, "zu untersuchen, wie die Unternehmenskontrolle durch gewerkschaftliche Repräsentanz auf Aufsichtsratsebene (`board of directors $\triangleleft$ ) radikal demokratisiert werden könne und welche Implikationen sich daraus für das Unternehmensrecht ergeben würden « $^{2}$, markierte einen tiefen Einschnitt in die bisherige Tradition einer weitgehend interventionsfreien Regulierung der Arbeitsbeziehungen und signalisierte eine Aufweichung der in der britischen Arbeiterbewegung tief verwurzelten Ablehnung rechtsverbindlicher Normen bei der Auseinandersetzung zwischen Beschäftigten und Unternehmern bzw. ihren Organisationen. Untrennbar verbunden mit diesen spezifisch unternehmens- und arbeitsrechtlichen Aspekten ist die Frage nach der Bedeutung und den Konsequenzen dieser Entwicklungstendenzen für die Strategie und Programmatik der britischen Gewerkschaften.

Dieser Beitrag will daher zunächst kurz die ökonomischen, politischen und arbeitsrechtlichen Rahmenbedingungen skizzieren, um dann die konkreten Konzeptionen für die Einführung gesetzlich verankerter Mitbestimmungsregelungen auf Unter-

\footnotetext{
I Department of Trade, $»$ Report of the Committee of Inquiry on Industrial Democracy *, Chairman Lord Bullock (im folgenden zitiert als "Bullock-Report $\%$ ), HMSO, Cmnd 6706, London January 1977
}

2 Ebd., S. V. 
nehmensebene im gesamtgesellschaftlichen Kontext diskutieren zu können. Abschließend soll eine vorläufige Einschätzung über die Realisierungsmöglichkeiten derartiger Vorstellungen versucht werden.

Die wirtschaftlichen und politischen Rabmenbedingungen

Mit dem Zerfall des englischen Kolonialreiches, dessen einstige Macht sich heute nur noch in der traditionsüberfrachteten und kostspieligen Fassade der britischen Königin als Staatsoberhaupt aller Commonwealth-Länder widerspiegelt, ist auch die britische Wirtschaft zunehmend in Schwierigkeiten geraten. Der Wegfall sowohl günstiger Absatzmärkte als auch billiger Rohstoffquellen führte zu hoher und immer steigender Auslandsverschuldung sowie zu ständigen Zahlungs- und Handelsbilanzkrisen und damit in der Folge zum Kursverfall des Pfund Sterling; gegenüber ausländischen Währungen, wie z. B. der DM, hat das britische Pfund allein im Verlauf der letzten sechs Jahre mehr als die Hälfte seines damaligen Wertes eingebüßt. Diese krisenhaften Tendenzen werden auch gegenwärtig vor allem durch zwei Momente verstärkt: Zum einen fließen weiterhin erhebliche Mittel für die Stationierung, Ausrüstung und Unterhaltung umfangreicher Streitkräfte in europäische Länder sowie vor allem in die überseeischen Kolonialgebiete. Zum anderen ist es insbesondere seit dem EG-Beitritt Großbritanniens im Jahre 1971 der private Kapitalexport, der zu einer entscheidenden Schwächung der inländischen Wirtschaft führt. Auch die im Inland durchaus noch in vielen Industriezweigen erwirtschafteten Gewinne werden nur ungenügend wieder re-investiert, fließen statt dessen z. B. ins Ausland ab, fehlen aber in jedem Fall zur Erneuerung bzw. Erweiterung der eigenen Industrie. Profitablen Beteiligungen in kontinentaleuropäischen Industrieund Handelszentren stehen fehlende Investitionen in die völlig überalterten Produktionsanlagen in Großbritannien gegenüber, die im Gegensatz zur Bundesrepublik nach dem Zweiten Weltkrieg eben nicht erneuert werden mußten und daher im Durchschnitt wesentlich älter, d. h. technologisch rückständiger, weniger effizient und somit weitaus unrationeller sind. Aus dem Kreislauf dieser Faktoren ergibt sich das zum Klischee gewordene Bild der »Englischen Krankheit«, die jedoch wider Erwarten noch immer nicht zum Ableben des chronisch Kranken geführt hat. Als gegenwärtig letztes Mittel wurde dem Patienten Ende 1976 vom Internationalen Währungsfond (IMF) mit der Auflage, mendlich Ordnung in Wirtschaft und Staat zu bringen «, eine Kreditspritze in Milliardenhöhe verabreicht, ohne daß jedoch absehbar wäre, daß damit die zugrundeliegenden strukturellen Schwächen des britischen Kapitalismus behoben werden könnten.

Vielmehr setzt sich die wirtschaftliche Dauerkrise Großbritanniens auch gegenwärtig unaufhaltsam fort. Im Juni I 977 lagen die Lebenshaltungskosten um 17,7\% höher als im selben Monat des Vorjahres, saisonal bereinigte Arbeitslosenstatistiken bedeuten mit 1,35 Millionen - das entspricht 5,9\% aller Beschäftigten - eine Rekordhöhe für die Zeit nach dem Zweiten Weltkrieg. Einschließlich der während der Sommerperiode hinzukommenden Schulabgänger betrug die Arbeitslosenquote bereits 6,8\%. Ende Oktober I 977 war die Zahl der Arbeitslosen bereits auf I 518375 gestiegen.

Ende des "Social Contract - Verlust der parlamentarischen Mebrheit

Kern- und Ausgangspunkt gegenwärtiger Krisenbewältigungsversuche war während der letzten drei Jahre der "Social Contract", der keinen rechtsverbindlichen Vertrag darstellt, sondern lediglich als ein programmatisches Konzept im Sinne einer gemeinsamen Absichtserklärung von den Spitzengremien des Gewerkschaftsdachverbandes, des Parteivorstandes und der Parlamentsfraktion der Labour Party 
vereinbart worden war. Dabei standen den gewerkschaftlichen Zugeständnissen für eine zurückhaltende Lohnpolitik die weitreichenden Zielsetzungen einer "grundlegenden Einkommens- und Vermögensumverteilung « sowie einer »ständigen Ausweitung der gewerkschaftlichen Aufgabenbereiche durch die Ausdehnung der Demokratisierung der Wirtschaft gegenüber “. ${ }^{3}$ Für die Beschäftigten war die Praxis in den letzten Jahren jedoch einseitig von den einkommenspolitischen Zugeständnissen geprägt: Trotz freiwilliger Lohnbeschränkungen i 975 (Obergrenze für Lohnerhöhungen $6 \&$ pro Woche) und 1976 (ca. $5 \%$ Lohnerhöhung mit festgelegter 2,50 £-Unter- und $4 \mathrm{f}$-Obergrenze pro Woche) blieben die Preissteigerungsraten zweistellig und führten für die Betroffenen zu erheblichen Reallohnverlusten und damit zu einem dauernden Absinken des allgemeinen Lebensstandards in Großbritannien. Als im Sommer 1977 eine weitere Phase freiwilliger Lohnbeschränkung vereinbart werden sollte, war es bezeichnenderweise der Jahreskongreß der Transportarbeitergewerkschaft TGWU - ihr Generalsekretär Jack Jones gilt aufgrund seines absoluten Loyalitätsanspruchs gegenüber der Labour-Regierung als »Architekt des Social Contract « -, der mit großer Mehrheit und gegen die Vorstandsempfehlung jede weitere Lohnbeschränkungs-Regelung ablehnte, statt dessen eine sofortige Rückkehr zum Prinzip freier Lohnverhandlungen beschloß und somit als mächtigste Einzelorganisation praktisch das vorläufige Ende staatlicher Einkommenspolitik besiegelte.

Der damit zum Ausdruck kommende Vertrauensschwund und die Enttäuschung über die Politik der Labour-Regierung, deren Sozialpakt-Praxis sich einseitig auf Krisenlösungsversuche zu Lasten der Beschäftigten beschränkt und demgegenüber die programmatischen Zusagen für wirtschafts- und gesellschaftspolitische Veränderungen nur bruchstückhaft verwirklicht hatte, schlug sich auch in den Parlamentsnachwahlen nieder und führte zum Verlust der ohnehin knappen Mehrheit im britischen Unterhaus. Gegenwärtig wird die politische Handlungsfähigkeit der Labour-Regierung nur noch durch Absprachen mit den Liberalen gewährleister, wobei auch die Uneinigkeit der verschiedenen schottischen, waliser und nordirischen Nationalisten im Parlament entscheidende Abstimmungsniederlagen und den endgültigen Sturz der Regierung verhindert haben.

\section{Entwicklung und gegenwärtige Situation des britischen Arbeitsrechts}

Das wesentlichste Merkmal der britischen Arbeitsbeziehungen, »industrial relations «, ist das Prinzip der Freiwilligkeit, dessen Kern immer die Erhaltung bzw. der Ausbau des "freien kollektiven Verhandlungswesens " (free collective bargaining ") gewesen ist. Damit ist gleichzeitig das Entscheidende über die Bedeutung des Rechts zur Regelung der kollektiven Beziehungen zwischen Beschäftigten und Unternehmern bzw. ihren jeweiligen Organisationen ausgesagt: Die industriellen Beziehungen sind in ihrer langen Entwicklungsgeschichte immer weitgehend von gesetzgeberischen Eingriffen ausgenommen geblieben. Bis in die jüngere Vergangenheit waren die gesetzlichen Regelungen immer bestimmt von negativen, gesetzlich verankerten Schutzbestimmungen, die den Verhandlungsparteien zwar »Freiheiten« zur Aktion, jedoch keine positiv verankerten »Rechte« zugestanden.

\footnotetext{
3 TUC-Labour Party Liaison Committee, "Economic Policy and the Cost of Living ", February 1973, S. 7; dieses Grundsatzpapier des gemeinsamen Verbindungskomitees war der Ausgangspunkt des Sozialpakts, ein von beiden Seiten unterzeichnetes Schriftstück mit dem Titel "Social Contract « hat es jedoch zu keiner Zeit gegeben. Der insbesondere für Ausländer irreführende Begriff "Social Contract “ - häufig mißverständlich übersetzt als »Sozialvertrag * tauchte dann erst 1974 in einem vom Gewerkschaftsdachverband TUC erstellten Programmpapier auf (TUC, "Collective Bargaining and the Social Contract ", 26. Juni 1974), das kurz darauf vom Jahreskongreß gebilligt wurde.
} 
Dies trifft auch für die historische Entwicklung der Gewerkschaften zu, die sich niemals auf ein positiv verankertes Recht auf Gewerkschaftsorganisationen berufen konnten, sondern deren gesetzliche Grundlage für eine legale Gewerkschaftsarbeit sich lediglich aus mehreren anderen "Negativ-Bestimmungen" ableiten läßt: 1824 - Aufhebung des Verbots für Vereinigungen zum Zweck gemeinsamer Interessenvertretung; 1871 - Aufhebung der Illegalisierung von Vereinigungen zur Behinderung von Handel und Geschäft; I875 - Aufhebung des generellen Streikverbots sowie der generellen Schadensersatzpflicht für Vereinigungen. Somit ist festzuhalten, daß der rechtliche Status einer Gewerkschaftsorganisation in Großbritannien zu keinem Zeitpunkt positiv und klar festgelegt und auch z. B. in bezug auf das Streikrecht formal niemals eine rechtliche Verankerung vorgenommen worden ist. Veränderungen in der Gesetzgebung hat es im wesentlichen nur deshalb gegeben, weil bei einzelnen Präzedenzfällen durch die Rechtsprechung stark abweichende Urteile gefällt und diese dann durch das Parlament wieder korrigiert wurden. Grundsätzlich blieb das Arbeitsrecht in Großbritannien nur ein Rahmenwerk der Nichteinmischung in die kollektiven Arbeitsbeziehungen. Auf seiten der abhängig Beschäftigten resultiert dieses historisch gewachsene Verständnis einer gewerkschaftlichen Unabhängigkeit aus der auch heute noch tiefen $\mathrm{K}$ luft zwischen »them and us«, d. h. zwischen den Unternehmern da oben und den Beschäftigten hier unten.

Der krasseste und für viele Kontinentaleuropäer wohl unverständlichste Unterschied bei der Regelung der Arbeitsbeziehungen liegt in der Handhabung von Lohn-, Arbeitszeit- und allen übrigen Abkommen: Es gibt in Großbritannien keine rechtsverbindlichen Abkommen wie z. B. die Tarifverträge in der Bundesrepublik. Dies bedeutet, daß alle Abmachungen und Vereinbarungen gleichsam als "gentlemen's agreement « in gutem Glauben ("in good faith «) oder als in Ehre verpflichtend ("binding in honour «) abgeschlossen werden. D. h., für beide Seiten besteht keine Möglichkeit einer gesetzlichen Einklagbarkeit; Veränderungen in den jeweiligen Voraussetzungen oder andere Gründe für eine Nichteinhaltung bzw. einen Abkommensbruch können nur durch unverzügliche und direkte Verhandlungen beider Seiten geregelt werden. Dabei sind die Verhandlungspositionen im wesentlichen bestimmt von der jeweiligen Verhandlungsstärke bzw. Durchsetzungskraft und nicht präjudiziert von rechtlich verbindlichen Rahmenbedingungen bzw. ihren jeweiligen juristischen Interpretationen. Insgesamt ist das System der Arbeitsbeziehungen in Großbritannien im Gegensatz zur Bundesrepublik eher als Kräfte- denn als Rechtsverhältnis zu bezeichnen.

Die gescheiterten Versuche von Labour- und Konservativen Regierungen in den sechziger Jahren, die wirtschaftlichen Krisen trotz freiwilliger und auch gesetzlich verordneter Lohnbeschränkungen auf Kosten der Beschäftigten zu lösen, führten dann zu den Konzepten, die starken und gesetzlich unkontrollierten Positionen der Gewerkschaften und ihrer wichtigsten Vertreter in den Betrieben, den "Shop Stewards «, in ein umfassendes Arbeitsrechtssystem einzuzwängen, um ihre Macht über rechtsverbindliche Vorschriften, Verbote, Straf- und Haftungsandrohungen entscheidend zu brechen bzw. besser kontrollieren zu können. Während das Labour-Konzept (1969/70) nach massivem Einspruch der Gewerkschaftsbewegung bereits im parlamentarischen Vorfeld scheiterte und die Enttäuschung über die

\footnotetext{
4 Vgl. hierzu ausführlich: Günther R. Degen, Shop Stewards - Ihre zentrale Bedeutung für die Gewerkschaftsbewegung in Großbritannien, Frankfurt/Köln 1976, S. 308-354.

s Vgl. ebd., insbesondere die systematische Analyse der gewerkschaftlichen Interessenvertretungsstrukturen, S. 166-295.
} 
Regierung unmittelbar in einen Wahlsieg der Konservativen mündete, wurde deren Arbeitsgesetz dann 1971 zwar verabschiedet, scheiterte jedoch in der Praxis an dem aktiven Widerstand der Gewerkschaftsbewegung. ${ }^{6}$

Als Reaktion auf diese seit dem Generalstreik 1926 schwersten sozialen Auseinandersetzungen kam es zu dem oben bereits angesprochenen Sozialpakt zwischen Labour-Party und Gewerkschaftsspitze. Der "Social Contract« bildete die "programmatische Neubegründung ${ }^{77}$ des historischen Bündnisses zwischen den $\mathrm{Ge}-$ werkschaften und der von ihnen im Gegensatz zu den Entstehungsprozessen kontinentaleuropäischer Arbeiterparteien bereits im Jahre I 899 gegründeten Labour Party. Auf dem Hintergrund dieser bisher erörterten gesamtpolitischen, ökonomischen und arbeitsrechtlichen Entwicklungsprozesse müssen auch die aktuellen Diskussionen und Auseinandersetzungen um unterschiedliche Demokratisierungskonzepte und ihre Durchsetzungsproblematiken analysiert und bewertet werden. So war eine der Grundvoraussetzungen für das Zustandekommen des "Social Contract« im Jahre 1972 die Verpflichtung der nächsten Labour-Regierung, »in der ersten Sitzungsperiode eine Gesetzesvorlage zur Zurücknahme des Arbeitsgesetzes (der Konservativen, d. V.) einzubringen. $\aleph^{8}$ Diese Zusage wurde in der Zeit von 1974 bis 1976 verwirklicht, indem mit dem neuen "Trade Union and Labour Relations Act (TULRA)《 die als »Anti-Streik-Bestimmungen« bekannt gewordenen Rechtsnormen - wie z. B. Verbot von spontanen Arbeitsniederlegungen, gerichtlich verfügte Streikurabstimmungen und gegebenenfalls Streikaufschubphasen (»Abkühlungsperioden ") sowie Verbot von "closed shop«-Abkommen, d. h. die ausschließliche Beschäftigung von gewerkschaftlich Organisierten in einem Betrieb und ferner die Rechtsverbindlichkeit von Abkommen zwischen Unternehmern und Gewerkschaften - abgeschafft und der status quo ante wiederhergestellt wurde.

Neben diesen Veränderungen im kollektiven Arbeitsrecht wurden im "Employment Protection Act 1975 " individuelle Rechte der Beschäftigten abgesichert, so u. a. Minimum-Lohngarantie bei Produktionsunterbrechungen, bezahlter Schwangerschaftsurlaub, längere Kündigungsfristen bei Entlassungen, Freistellung für innerbetriebliche Gewerkschaftstätigkeiten sowie für gewerkschaftliche Bildungsmaßnahmen. Darüber hinaus wurden erstmals Bestimmungen gesetzlich verankert, durch die Unternehmen zur Anerkennung gewerkschaftlicher Organisation und Repräsentation sowie zur Bereitstellung von Unternehmensinformationen für die Gewerkschaften verpflichtet werden. Weiterhin ist noch der »Health and Safety at Work Act 1974« zu nennen, durch den die Uberwachung aller bisherigen Arbeitsplatzsicherheitsbestimmungen zentralisiert wird und ferner das ausdrückliche Recht auf gewerkschaftliche Sicherheitsbeauftragte in jedem Betrieb und deren Freistellung für ihre Aufgaben und ihre Weiterbildung gesetzlich verankert worden ist.

Die Ergebnisse der Bullock-Komission: »2X+Y*

Zur Verwirklichung des programmatischen Anspruchs einer grundlegenden und umfassenden Demokratisierung der Wirtschaft hatte die Labour-Regierung die eingangs erwähnte "Bullock-Komission « eingesetzt, die die verschiedenen Modelle und Konzeptionen einer Arbeitnehmerbeteiligung auf Unternehmensebene untersuchte und Anfang 1977 in ihrem Bericht konkrete Vorschläge vorlegte:

- An der Unternehmensspitze jeweils ein eingleisiges Geschäfführungs- und Kon-

6 Vgl. ebd., insbesondere das Kapitel "Industrial Relations Act 197r - Analyse der Praxis«, S. 355-393; vgl. dazu ferner U. Mückenberger, Arbeitsrecht und Klassenkampf, Frankfurt/Köln 1974.

7 Günther R. Degen, „Gewerkschaften in Großbritannien - Sozialpakt und Demokratisierungskonzepte als Eckpfeiler gewerkschaftlicher Programmatik ", in: Gewerkschaftliche Monatshefte 9/1976, S. $53 \mathrm{I} \mathrm{ff.}$ 8 TUC-Labour Party Liaison Committee, "Statement, 26 July 1972*. 
trollgremium (»board of directors «), das nach der Formel $2 \mathrm{X}+\mathrm{Y}$ zusammengesetzt werden soll. Mit $2 \mathrm{X}$ ist eine gleichstarke Anzahl von Vertretern der Anteilseigner $\left(\mathrm{X}_{1}\right)$ und der Beschäftigten $\left(\mathrm{X}_{2}\right)$ gemeint, die sich dann gemeinsam auf eine kleinere dritte Gruppe $(\mathrm{Y})$ mit ungerader Personenzahl einigen soll. Für diese dritte Gruppe kommen ausschließlich betriebsfremde Personen in Frage, es können z. B. sowohl Unternehmensverbandsvertreter als auch hauptamtliche Gewerkschaftsfunktionäre sowie Finanz- oder Wirtschaftsfachleute etc. sein.

- Als Geltungsbereich für ein derartiges Beteiligungsmodell sind alle privatwirtschaftlichen Unternehmen mit mehr als 2000 Beschäftigten vorgesehen. Gegenwärtig wären dies in Großbritannien 738 Unternehmensgruppen mit etwa 2000 Betrieben und ca. 7 Millionen Beschäftigten.

- Die Einführung solcher neu zusammengesetzter Unternehmensführungen soll jedoch nicht für alle betroffenen Unternehmen gesetzlich verbindlich vorgeschrieben werden, sondern von einer Mehrheitsentscheidung der jeweiligen Belegschaften abhängig gemacht werden. Beantragt werden kann eine derartige Abstimmung von einer Gewerkschaft, die $20 \%$ der betroffenen Beschäftigten repräsentiert. Bei der Abstimmung reicht eine einfache Mehrheit der abgegebenen Stimmen, wenn diese mindestens ein Drittel der Gesamtbelegschaft ausmacht.

- Eine daraufhin möglich werdende Wahl der Arbeitnehmer-Vertreter soll ausschließlich auf gewerkschaftlicher Basis erfolgen, d. h. wahlberechtigt sind nur die gewerkschaftlich Organisierten. Die im Betrieb vertretenen Gewerkschaften sollen auch in der Frage von Wahlordnung und -verfahren frei sein, die Kommission empfiehlt sogar ausdrücklich, daß die Arbeitnehmer-Vertreter aus den betrieblichen Shop Steward-Vertretungen hervorgehen und damit grundsätzlich Betriebsangehörige sein sollen. Eine Abwahlmöglichkeit im Verlauf der vorgeschlagenen dreijährigen Amtszeit wird ebenfalls eingeräumt, und zwar für den Fall, daß alle vertretenen Gewerkschaften durch ihre autorisierten Sprecher im Betrieb dies gemeinsam befinden.

Vor einer grundsätzlichen Einordnung des Gesamtkonzepts der Bullock-Kommission sollen die dargelegten Vorschläge im einzelnen kurz auf ihre Bedeutung hin untersucht werden. Die Formel $» 2 \mathrm{X}+\mathrm{Y}$ « für die Zusammensetzung der jeweiligen Unternehmensführung stellt den Versuch dar, den Beschäftigten im Sinne einer "Parität« die gleiche Anzahl von Vertretern wie den Aktionären im »board of directors" zu gewährleisten; diese z. B. jeweils sieben Anteilseigner- bzw. Belegschaftsvertreter müßten sich dann noch auf fünf weitere, außerbetriebliche Mitglieder einigen. Dieses Konzept wird ausdrücklich als Modifizierung kontinentaleuropäischer Mitbestimmungsmodelle verstanden, wobei im Vergleich zur Bundesrepublik die bei uns getrennten Aufsichtsrats- und Vorstandsebenen vereinheitlicht und die Position des »Neutralen« aus der Montanmitbestimmung lediglich zahlenmäßig erweitert werden soll. Der modellhafte und schematische Übertragungsversuch auf Großbritannien wird auch darin deutlich, daß die Kommission auf mögliche Einigungs- und Entscheidungskonflikte weder in bezug auf die Konstituierung noch auf konkrete Sachauseinandersetzungen eingeht, sondern die vorgeschlagene Formel allein als ausreichende Voraussetzung für eine im wesentlichen konfliktfreie Entscheidungsfindung anzusehen scheint.

In bezug auf den Geltungsbereich muß kritisch angemerkt werden, daß lediglich das Kriterium der Beschäftigtenzahl zugrundegelegt werden soll. Damit würden diejenigen wirtschaftlich wichtigen Konzerne aus dem vorgesehenen Geltungsbereich herausfallen, die als kapital- und wenig personalintensive Unternehmen zwar möglicherweise unter 2000 Beschäftigte, gemessen an ihrem Umsatz jedoch außerordentliche wirtschaftliche Bedeutung haben. Die geplante mehrheitliche Abstimmung der 
betroffenen Belegschaft als Voraussetzung für die Einführung des Beteiligungsmodells sowie insbesondere der dafür dann vorgesehene Wahlmodus sind deutliche Hinweise auf eine konsequente Anbindung und Einbettung der gesamten Konzeption in die gewerkschaftlichen Interessenvertretungsstrukturen. So kann z. B. die Initiativkraft nur von den betrieblich vertretenen Gewerkschaften ausgehen, wobei an der eigentlichen Abstimmung dann zwar noch alle Beschäftigten teilnehmen können, bei der dann folgenden Vertreter-Wahl jedoch ausschließlich die gewerkschaftlich organisierten Belegschaftsmitglieder das aktive und passive Wahlrecht ausüben dürfen.

Die Kommission bemühte sich, durch weitere Vorschläge - wie der dauernden und umfassenden Rückkopplung zwischen Unternehmens- und Betriebsebene sowie durch die ausdrückliche Abwahlmöglichkeit der Arbeitnehmervertreter durch die gewerkschaftlich Organisierten - einen eindeutigen Gewerkschaftsbezug zu betonen. Dennoch wird bei der Funktions- und Verantwortungsbestimmung für die board-Mitglieder insgesamt das bisherige Unternehmensrecht im Kern nicht angetastet. Danach sollen alle Direktoren gesetzlich verpflichtet sein, "sowohl die Interessen aller im Unternehmen Beschäftigten als auch die Interessen der Anteilseigner wahrzunehmen «.9 Mit einer derartigen Festlegung wird der zuvor angedeutete Ansatz einer konsequenten Verzahnung mit den gewerkschaftlichen Interessenvertretungen im Betrieb entscheidend relativiert, indem die Arbeitnehmervertreter auf Unternehmensebene auf das Gewinninteresse der Aktionäre verpflichtet und damit in eine Zwitterfunktion zu Lasten ihrer ursprünglichen Aufgabenstellung als Belegschaftsvertreter gedrängt werden. Die Kommission unterstreicht an anderer Stelle des Berichts dieses entscheidend eingeschränkte Verständnis über die Rolle der betrieblichen Mitbestimmungsträger. Danach sollen sie "gewählte Vertreter (rrepresentatives ) sein, frei in ihrer Meinungsäußerung und Entscheidungsfindung, welche Maßnahmen zum größeren Wohl des Betriebes beitragen werden, und nicht Delegierte (’delegates ), die gemäß des ausdrücklichen Willens ihrer Wähler abstimmen «. ${ }^{\text {IO }}$

Diese Charakterisierung bedeutet eine Absage an das dominierende Prinzip der gewerkschaftlichen Interessenvertretung im Betrieb, das eben gerade auf einer direkten Verbindung und wechselseitigen Abhängigkeit zwischen den gewerkschaftlich Organisierten und ihren gewählten Vertretern - den Shop Stewards - beruht. Auch auf dem Hintergrund der eingangs skizzierten Entwicklungsprozesse und Schwerpunktsetzungen in der britischen Arbeiterbewegung bedeuten die erörterten Konzeptionen für eine gewerkschaftliche Beteiligung an der privatwirtschaftlichen Unternehmensführung eine krasse Abkehr vom bisherigen Selbstverständnis und der dementsprechenden Praxis. Denn traditionell wurde von der britischen Gewerkschaftsbewegung eine Beteiligung und Verantwortung bei Unternehmensentscheidungen immer konsequent abgelehnt. Statt dessen stand der Kampf um die Erhaltung und Ausweitung einer autonomen und nicht von staatlichen und rechtlichen Interventionen beeinflußten Interessenvertretungsarbeit mit eindeutiger, unmittelbar betrieblicher Schwerpunktsetzung immer im Vordergrund. " Insgesamt müssen die Pläne für eine rechtlich kodifizierte Beteiligung der Beschäftigten an der Unternehmensführung einerseits klar als einschneidender Bruch in der weit über einhundertjährigen Geschichte der britischen Arbeiterbewegung bewertet werden. Andererseits lassen die in Großbritannien erst in jüngster Zeit in Gang gekommenen Diskussionen - die wesentlich durch kontinentaleuropäische Mitbestimmungsmo-

$9 \mathrm{Vg}$. Companies Bill 1973, Clause 53 sowie Industrial Democracy Bill 1975, Clause I.

10 Vgl. "Bullock-Report«, a. a. O., S. 8s. 
delle und insbesondere durch die Bestrebungen für ein so orientiertes, vereinheitlichtes Europäisches Unternehmensrecht initiiert worden sind - erkennen, daß die bisher einseitig betrieblich ausgerichtete Gewerkschaftsarbeit aufgrund der ökonomischen Krisenentwicklung und im Hinblick auf die zunehmende internationale Kapital- und Unternehmensverflechtung allein als nicht mehr ausreichend zur Verteidigung bzw. Verbesserung der Arbeits- und Lebensbedingungen eingeschätzt wird. Gerade die gegenwärtigen, kontroversen Diskussionen über die Kommissionsergebnisse von Bullock machen innerhalb der Gewerkschaftsbewegung deutlich, wie sehr man zwar um eine Fortentwicklung der eigenen Strategievorstellungen bemüht ist, wie gering jedoch in bezug auf eine rechtlich formalisierte Unternehmensmitbestimmung das Informationsniveau, die notwendigen Beurteilungsmaßstäbe sowie insbesondere die eigenen Erfahrungswerte ausgeprägt bzw. bisher überhaupt nicht vorhanden sind.

\section{Einschätzungen, Realisierungsmöglichkeiten, Perspektiven}

Die Reaktionen innerhalb der britischen Gewerkschaftsbewegung waren außerordentlich kontrovers. Der Gewerkschaftsdachverband TUC, der seit 1973 eine Aufsichtsratsstruktur mit paritätischer Gewerkschaftsbeteiligung nach bundesrepublikanischem Vorbild favorisiert, ${ }^{12}$ lobte den grundsätzlichen Ansatz einer ausdrücklich gewerkschaftlich eingebetteten Beteiligung auf Unternehmensebene. In einer Stellungnahme äußerte der Vorsitzende, Len Murray, die Uberzeugung, daß die Behebung der wirtschaftlichen Schwierigkeiten nur durch eine gemeinsame Planung der Unternehmensstrategien erreicht werden könne, "die entscheidend abhängig von der Schaffung einer neuen, durch Gleichheit und Kooperation geprägten Beziehung zwischen Kapital und Arbeit sei«.' ${ }^{13}$ Der Vorsitzende der größten Einzelgewerkschaft, der Transportarbeitergewerkschaft TGWU, Jack Jones, schätzt die Arbeit der Bullock-Kommission positiv als ersten Schritt zur Verwirklichung der programmatischen Ansprüche des "Social Contract « ein und fordert auf dieser Grundlage die günstige Gelegenheit zu nutzen, »um den Worten nun Taten folgen zu lassen «. ${ }^{4}$

Demgegenüber steht eine vielschichtige Kritik an dem grundsätzlichen Ansatz einer formalisierten und gesetzlich verankerten Unternehmensmitbestimmung. Der Vorsitzende der zweitgrößten Einzelgewerkschaft, des Metallarbeiterverbandes AUEW, Hugh Scanlon, lehnt Beteiligungsmodelle in der Privatwirtschaft grundsätzlich ab und fordert die Verwirklichung der Demokratisierungsziele allein durch eine Ausweitung des interventionsfreien Verhandlungswesens. 's Scharf kritisiert auch der Vorsitzende der drittgrößten Organisation, der Allgemeinen Arbeiter- und Kommunalbediensteten-Gewerkschaft GMWU, David Basnett, die Kommissionsvorstellungen, die er für viel zu schematisch hält, um den unterschiedlichen Bedingungen in den einzelnen Industriezweigen und Betrieben gerecht werden zu kön-

1 I Vgl. Günther R. Degen, „Das Dilemma der britischen Gewerkschaften: Gewerkschaftliche Kampfkraft zwischen betrieblicher Stärke und gesamtgesellschaftlicher Ohnmacht«, in: J. Bergmann (Hrsg.), Soziologie der Gewerkschaften (erscheint voraussichtlich Ende 1977 in der edition suhrkamp).

$12 \mathrm{Vgl}$. Fußnote 7; bezeichnenderweise geht der britische Gewerkschaftsdachverband TUC davon aus, daß die Mängel einer fehlenden gewerkschaftlichen Verzahnung bei den bundesrepublikanischen Betriebsverfassungs- und Mitbestimmungsregelungen durch die TUC-Konzeption ausgeschaitet seien. So betonte der Vorsitzende Len Murray auf dem Jahreskongreß 1976: "Es gab einmal einen großen Philosophen, der die Notwendigkeit betonte, Hegel auf die Füße zu stellen. Nun, wir haben das deutsche Konzept . . . auf die Füße gestellt.

13 TUC, "TUC Guide to the Bullock Report on Industrial Democracy “, London Februar 1977, S. 5.

I 4 "The Record «, Monatszeitschrift der TGWU, Februar 1977.

is Vgl. The Times, 27. I. 1977. 
nen. ${ }^{16}$ Die politisch äußerst gemäßigte, sechstgrößte Elektriker- und Klempnergewerkschaft EEPTU begründet ihre Ablehnung mit dem klassischen Argument der britischen Gewerkschaften: Es sei Aufgabe einer gewerkschaftlichen Interessenvertretung, »die unternehmerische Entscheidungsgewalt in Frage zu stellen, falls notwendig, ihre Anwendung zu bekämpfen. Es ist nicht die Verantwortung der abhängig Beschäftigten, ein Unternehmen zu leiten, vielmehr ist es unabdingbar, daß die Gewerkschaften ihre Unabhängigkeit bewahren. «' Eine weitere Reaktion ist kennzeichnend für die ablehnende Einschätzung vieler Einzelgewerkschaften. So erklärte auf dem Jahreskongreß des Dachverbandes ein Vertreter der Gewerkschaft Leitender Postangestellter: "Wir werden in Wirklichkeit als trojanisches Pferd vom Unternehmen gebraucht, um unsere Mitglieder zur Zustimmung zu solchen Maßnahmen zu bewegen, von denen das Unternehmen weiß, daß es eine derartige Zustimmung auf dem Weg normaler Kollektivverhandlungen niemals bekommen hätte. ${ }^{18}$ Die kontroversen, in der Mehrzahl jedoch negativen Reaktionen auf die Bullock-Vorschläge - der Vollständigkeit halber sei hier noch die kraß ablehnende Haltung von seiten der Unternehmer erwähnt ${ }^{19}$ - können in einem Teil bereits die Frage nach den Realisierungsmöglichkeiten beantworten. Die bisherigen Diskussionen stehen zwar erst am Anfang, grundsätzlich sind jedoch starke Vorbehalte innerhalb der Gewerkschaftsbewegung festzustellen.

Unabhängig von den gewerkschaftlichen Diskussionen ist die Verabschiedung derartiger Mitbestimmungsgesetze in Großbritannien politisch gegenwärtig nahezu ausgeschlossen. Nach dem Verlust mehrerer Unterhaus-Nachwahlen sowie der Ablehnung weiterer freiwilliger Lohnverzichtsabkommen durch die Gewerkschaften ist die Labour-Regierung in wichtigen wirtschafts- und gesellschaftspolitischen Entscheidungen nicht mehr allein handlungsfähig, sondern nach dem Verlust der absoluten Mehrheit ständig von Absprachen mit den Liberalen abhängig. Wenn überhaupt, so wird die jetzige Labour-Regierung vor Neuwahlen nur noch verstärkt versuchen, Demokratisierungskonzepte in den verstaatlichten Sektoren durchzusetzen, um wenigstens einen Bruchteil der im "Social Contract « formulierten Ansprüche zu realisieren. In diesem Zusammenhang wurde bereits Ende Juli 1977 für den Bereich der Post ein Gesetz verabschiedet, ${ }^{20}$ auf dessen Grundlage gegenwärtig ein zunächst auf zwei Jahre befristetes Experiment vorbereitet wird: Das oberste Leitungs- und Führungsgremium soll danach aus jeweils sieben Vertretern des Managements bzw. der vertretenen Gewerkschaften sowie fünf Unabhängigen zusammengesetzt werden, wobei zwei Personen dieser außerbetrieblichen Vertreter ausdrücklich die Verbraucherinteressen repräsentieren sollen. Die Vorbereitung dieses Modellversuchs im verstaatlichten Postbereich findet unter Mitwirkung der in diesem Sektor vertretenen Gewerkschaften statt und macht deutlich, daß ein Teil der Einzelgewerkschaften - so z. B. der Metallarbeiterverband AUEW - eine differenzierte Auffassung vertreten und bei den verstaatlichten Industrien durchaus Modelle einer formalisierten Mitbestimmung auf Unternehmensebene befürworten.

Aus der bisherigen Erörterung der Konzeptionen für eine gesetzlich verankerte Mitbestimmung auf Unternehmensebene scheint erkennbar zu sein, daß sich kurz-

\footnotetext{
i6 Vgl. ebd., sowie The Times 2. 2. 1977.

17 „EEPTU Evidence», paragraph 24 ff., zit. nach: "Bullock-Report«, a. a. O., S. I 24.

18 Zit. nach: TUC Report 1975, S. 540.

19 Dies hatte auch bereits in einem Minderheiten-Votum dreier Kommissionsmitglieder seinen Niederschlag gefunden, die in einem "Minority Report« als Anhang ihre ablehnende Haltung begründen: Vgl. "Bullock-Report", a. a. O., S. 167-195.

20 "Post Office Act 1977. 29. Juli 1977٪, HMSO, London 1977.
} 
fristig und unmittelbar daraus keine Perspektiven für die britische Gewerkschaftsbewegung ableiten und realisieren lassen. Ein wichtiger positiver Aspekt muß jedoch darin gesehen werden, daß über die zunächst im Ansatz nur von außen herangetragenen Vorstellungen weiterführender gewerkschaftlicher Strategievorstellungen in Großbritannien erstmals eine breitere Diskussion und Reflexion der eigenen Gewerkschaftsarbeit in Gang gekommen ist. Die konkrete Auseinandersetzung mit der eigenen Praxis sowie mit grundlegend anderen Ansätzen ist nicht nur zur eigenen Positionsbestimmung und deren Weiterentwicklung notwendig, sondern leistet damit auch die unabdingbare Voraussetzung für eine immer wieder geforderte, jedoch bis heute noch nicht wesentlich konkretisierte bzw. realisierte internationale Gewerkschaftsstrategie gegen die ständig wachsende Macht multinationaler Unternehmen.

Güntber R. Degen

\section{Aufruf zum Stand der Freiheitsrechte in der Bundes- republik Deutschland}

Gemeinsames Kommuniqué des Syndicat de la Magistrature und der Magistratura Democratica vom 26. Oktober 1977

(Deutsche Obersetzung)

Angesichts der Entwicklung der demokratischen Freiheitsrechte in der BRD haben Organisationen italienischer und französischer Richter und Staatsanwälte, Magistratura Democratica und das Syndicat de la Magistrature, beschlossen, einen gemeinsamen Aufruf zu veröffentlichen:

Sie stellen fest, daß in der BRD die fundamentalen Persönlichkeitsrechte, die sowohl im Grundgesetz als auch in der Allgemeinen Menschenrechtserklärung und in der Europäischen Menschenrechtskonvention verankert sind, schrittweise unter dem Vorwand des Kampfs gegen eine politische Protestbewegung eingeschränkt werden, die allzuoft mit terroristischen Randerscheinungen identifiziert wird.

Sie verurteilen die Maßnahmen, die zur Bekämpfung des politischen Protests ergriffen werden, insbesondere:

- die Berufsverbote;

- die Haftbedingungen der politischen Gefangenen, die der totalen Isolation und der Willkür des Machtapparats ausgeliefert sind;

- den Ausschluß der Anwälte und der Angeklagten selbst von einigen Prozessen;

- das Abhören der Telefone und die Durchsuchungen in Anwaltsbüros, die standes- und strafrechtlichen Verfolgungen einiger Anwälte, alles Praktiken, die Verletzungen der Rechte der Verteidigung darstellen;

- die Aufrufe zur allgemeinen Denunziation, die außer Kontrolle zu geraten drohen und ein Klima schaffen, in dem Gewalt und gegenseitige Verdächtigungen um sich greifen;

- die Psychose, die jeden politischen Protest als >extremistisch diffamiert und ihn mit dem Terrorismus oder seiner Unterstützung gleichstellt.

Sie rufen in Erinnerung, daß auf der einen Seite nichts, und sei es die sogenannte Staatsraison selbst, derartige Einschränkungen der Grundrechte zu rechtfertigen 